\title{
Controllability of Neutral Fractional Functional Equations with Impulses and Infinite Delay
}

\author{
R. Ganesh, ${ }^{1}$ R. Sakthivel, ${ }^{2}$ Yong Ren, ${ }^{3}$ S. M. Anthoni, ${ }^{1}$ and N. I. Mahmudov ${ }^{4}$ \\ ${ }^{1}$ Department of Mathematics, Anna University, Regional Centre, Coimbatore 641 047, India \\ ${ }^{2}$ Department of Mathematics, Sungkyunkwan University, Suwon 440-746, Republic of Korea \\ ${ }^{3}$ Department of Mathematics, Anhui Normal University, Wuhu 241000, China \\ ${ }^{4}$ Department of Mathematics, Eastern Mediterranean University, Gazimagusa, Mersin 10, Turkey
}

Correspondence should be addressed to R. Sakthivel; krsakthivel@yahoo.com

Received 15 March 2013; Accepted 11 July 2013

Academic Editor: Abdelaziz Rhandi

Copyright (C) 2013 R. Ganesh et al. This is an open access article distributed under the Creative Commons Attribution License, which permits unrestricted use, distribution, and reproduction in any medium, provided the original work is properly cited.

\begin{abstract}
We examine the controllability problem for a class of neutral fractional integrodifferential equations with impulses and infinite delay. More precisely, a set of sufficient conditions are derived for the exact controllability of nonlinear neutral impulsive fractional functional equation with infinite delay. Further, as a corollary, approximate controllability result is discussed by assuming compactness conditions on solution operator. The results are established by using solution operator, fractional calculations, and fixed point techniques. In particular, the controllability of nonlinear fractional control systems is established under the assumption that the corresponding linear control system is controllable. Finally, an example is given to illustrate the obtained theory.
\end{abstract}

\section{Introduction}

Control theory is an area of application-oriented mathematics which deals with the analysis and design of control systems. In particular, the concept of controllability plays an important role in various areas of science and engineering. More precisely, the problem of controllability deals with the existence of a control function, which steers the solution of the system from its initial state to a final state, where the initial and final states may vary over the entire space. Control problems for various types of deterministic and stochastic dynamical systems in infinite dimensional systems have been studied in [1-6].

On the other hand, the impulsive differential systems can be used to model processes which are subject to abrupt changes, and which cannot be described by the classical differential systems [7]. Moreover, impulsive control which is based on the theory of impulsive equations, has gained renewed interests due its promising applications towards controlling systems exhibiting chaotic behavior. Therefore, the controllability problem for impulsive differential and integrodifferential systems in Banach spaces has been studied extensively (see [8] and the references therein). Moreover, fractional calculus has received great attention, because fractional derivatives provide an excellent tool for the description of memory and hereditary properties of various processes [9]. Also, the study of fractional differential equations has emerged as a new branch of applied mathematics, which has been used for construction and analysis of mathematical models in various fields of science and engineering [10]. Therefore, the problem of the existence of solutions for various kinds of fractional differential systems has been investigated in [11-13]. Very recently, Dabas and Chauhan [14] studied the existence, uniqueness, and continuous dependence of mild solution for an impulsive neutral fractional order differential equation with infinite delay by using the fixed point technique and solution operator on a complex Banach space.

Recently, many authors pay their attention to study the controllability of fractional evolution systems $[15,16]$. Wang and Zhou [17] investigated the complete controllability 
of fractional evolution systems without involving the compactness of characteristic solution operators. Kumar and Sukavanam [18] derived a new set of sufficient conditions for the approximate controllability of a class of semilinear delay control systems of fractional order by using contraction principle and the Schauder fixed point theorem. Sakthivel et al. [19] studied the controllability results for a class of fractional neutral control systems with the help of semigroup theory and fixed point argument. The minimum energy control problem for infinite-dimensional fractional-discrete time linear systems is discussed in [20]. Debbouche and Baleanu [21] derived a set of sufficient conditions for the controllability of a class of fractional evolution nonlocal impulsive quasilinear delay integrodifferential systems by using the theory of fractional calculus and fixed point technique.

However, controllability of impulsive fractional integrodifferential equations with infinite delay has not been studied via the theory of solution operator. Motivated by this consideration, in this paper, we investigate the exact controllability of a class of fractional order neutral integrodifferential equations with impulses and infinite delay in the following form:

$$
\begin{gathered}
D_{t}^{q}\left[x(t)+g\left(t, x_{t}\right)\right]=A\left[x(t)+g\left(t, x_{t}\right)\right] \\
+J_{t}^{1-q}\left[B u(t)+f\left(t, x_{t}, H x(t)\right)\right], \\
t \in J=[0, b], \quad t \neq t_{k}, \\
\Delta x\left(t_{k}\right)=I_{k}\left(x\left(t_{k}^{-}\right)\right), \quad k=1,2, \ldots, m, \\
x_{0}=\phi \in \mathscr{B}_{h},
\end{gathered}
$$

where $D_{t}^{q}$ is the Caputo fractional derivative of order $q, 0<$ $q<1 ; A: D(A) \subset X \rightarrow X$ is an infinitesimal generator of the solution operator, $\left\{S_{q}(t)\right\}_{t \geq 0}$ is defined on a Banach space $X$ with the norm $\|\cdot\|_{X}$; the control function $u(\cdot)$ is given in $L^{2}(J, U), U$ is a Banach space; $B$ is a bounded linear operator from $U$ into $X$; the histories $x_{t}:(-\infty, 0] \rightarrow X$ are defined by $x_{t}(\theta)=x(t+\theta)$ belongs to an abstract phase space $\mathscr{B}_{h}$ defined axiomatically, and $I_{k}: X \rightarrow X, k=1,2, \ldots, m$ are bounded functions. Also, the fixed times $t_{k}$ satisfy $0 \leq t_{0}<$ $t_{1}<t_{2}<\cdots<t_{m}<t_{m+1} \leq b, \Delta x\left(t_{k}\right)=x\left(t_{k}^{+}\right)-x\left(t_{k}^{-}\right)$, and $x\left(t_{k}^{+}\right)=\lim _{h \rightarrow 0} x\left(t_{k}+h\right)$ and $x\left(t_{k}^{-}\right)=\lim _{h \rightarrow 0} x\left(t_{k}-h\right)$ denote the right and left limits of $x(t)$ at $t=t_{k}$, respectively. Further, $g: J \times \mathscr{B}_{h} \rightarrow X, f: J \times \mathscr{B}_{h} \times X \rightarrow X$ are given functions; the term $H x(t)$ is given by $H x(t)=$ $\int_{0}^{t} G(t, s) x(s) d s$, where $G \in C\left(D, R^{+}\right)$is the set of all positive continuous functions on $D=\left\{(t, s) \in R^{2}: 0 \leq s \leq t \leq b\right\}$. The main aim of this paper is to obtain some suitable sufficient conditions for the controllability results corresponding to admissible control sets without assuming the semigroup is compact. Further, we address the approximate controllability issue for the considered fractional systems. In order to prove the controllability results, we follow a technique similar to that of $[14,19]$ with some necessary modifications.

\section{Preliminaries}

In this section, we will recall some basic definitions and lemmas which will be used in this paper. Let $L(X)$ denote the Banach space of bounded linear operators from $X$ into $X$ with the norm $\|\cdot\|_{L(X)}$. Let $C(J, X)$ denote the space of all continuous functions from $J$ into $X$ with the norm $\|x\|=$ $\sup _{t \in J}\|x(t)\|$.

Now, we present the abstract space $\mathscr{B}_{h}$ [7]. Let $h$ : $(-\infty, 0] \rightarrow(0,+\infty)$ be a continuous function with $l=$ $\int_{-\infty}^{0} h(t) d t<+\infty$. For any $a>0$, define $\mathscr{B}=\{\varphi:$ $[-a, 0] \rightarrow X$ such that $\varphi(t)$ is bounded and measurable $\}$ and equip the space $\mathscr{B}$ with the norm $\|\varphi\|_{[-a, 0]}=$ $\sup _{s \in[-a, 0]}\|\varphi(s)\|, \varphi \in \mathscr{B}$. Further, define the space $\mathscr{B}_{h}=$ $\left\{\varphi:(-\infty, 0] \rightarrow X\right.$, for any $c>0,\left.\varphi\right|_{[-c, 0]} \in \mathscr{B}$ with $\varphi(0)=$ 0 and $\left.\int_{-\infty}^{0} h(s)\|\varphi\|_{[s, 0]} d s<+\infty\right\}$. If $\mathscr{B}_{h}$ is endowed with the norm $\|\varphi\|_{\mathscr{B}_{h}}=\int_{-\infty}^{0} h(s)\|\varphi\|_{[s, 0]} d s, \varphi \in \mathscr{B}_{h}$, then $\left(\mathscr{B}_{h},\|\cdot\|_{\mathscr{B}_{h}}\right)$ is a Banach space.

We assume that the phase space $\left(\mathscr{B}_{h},\|\cdot\|_{\mathscr{B}_{h}}\right)$ is a seminormed linear space of functions mapping $(-\infty, 0]$ into $X$ and satisfying the following fundamental axioms [22].

(A1) If $x:(-\infty, b] \rightarrow X, b>0$, is continuous on $J$ and $x_{0} \in \mathscr{B}_{h}$, then for every $t \in J$, the following conditions hold:

(i) $x_{t} \in \mathscr{B}_{h}$,

(ii) $\|x(t)\| \leq L\left\|x_{t}\right\|_{\mathscr{B}_{h}}$,

(iii) $\left\|x_{t}\right\|_{\mathscr{B}_{h}} \leq C_{1}(t) \sup _{0 \leq s \leq t}\|x(s)\|+C_{2}(t)\left\|x_{0}\right\|_{\mathscr{B}_{h}}$, where $L>0$ is a constant; $C_{1}:[0, b] \rightarrow[0, \infty)$ is continuous, $C_{2}:[0, \infty) \rightarrow[0, \infty)$ is locally bounded, and $C_{1}, C_{2}$ are independent of $x(\cdot)$.

(A2) For the function $x(\cdot)$ in (A1), $x_{t}$ is a $\mathscr{B}_{h}$-valued function on $[0, b]$.

(A3) The space $\mathscr{B}_{h}$ is complete.

Definition 1 (see [10]). The Caputo derivative of order $q$ for a function $f:[0, \infty) \rightarrow R$ can be written as

$$
D_{t}^{q} f(t)=\frac{1}{\Gamma(n-q)} \int_{0}^{t}(t-s)^{n-q-1} f^{(n)}(s) d s=I^{n-q} f^{n}(t)
$$

for $n-1<q<n, n \in N$. If $0<q \leq 1$, then

$$
D_{t}^{q} f(t)=\frac{1}{\Gamma(1-q)} \int_{0}^{t}(t-s)^{-q} f^{(1)}(s) d s .
$$

The Laplace transform of the Caputo derivative of order $q>0$ is given as

$$
\begin{array}{r}
L\left\{D_{t}^{q} f(t): \lambda\right\}=\lambda^{q} \widehat{f}(\lambda)-\sum_{k=0}^{n-1} \lambda^{q-k-1} f^{(k)}(0) ; \\
n-1<q<n .
\end{array}
$$


The Mittag-Leffler type function in two arguments is defined by the series expansion

$$
\begin{array}{r}
E_{q, p}(z)=\sum_{k=0}^{\infty} \frac{z^{k}}{\Gamma(q k+p)}=\frac{1}{2 \pi i} \int_{C} \frac{\mu^{q-p} e^{\mu}}{\mu^{q}-z} d \mu \\
q, p>0, z \in \mathbb{C}
\end{array}
$$

where $C$ is a contour which starts and ends at $-\infty$ and encircles the disc $\|\mu\| \leq|z|^{1 / 2}$ counter clockwise. The Laplace transform of the Mittag-Leffler function is given as follows:

$$
\begin{array}{r}
\int_{0}^{\infty} e^{-\lambda t} t^{p-1} E_{q, p}\left(\omega t^{q}\right) d t=\frac{\lambda^{q-p}}{\lambda^{q}-\omega}, \\
\operatorname{Re} \lambda>\omega^{1 / q}, \quad \omega>0
\end{array}
$$

and for more details (see [14]).

Definition 2 (see [9]). A closed and linear operator $A$ is said to be sectorial if there are constants $\omega \in R, \theta \in$ $[\pi / 2, \pi]$, and $M>0$, such that the following two conditions are satisfied:

(i) $\rho(A) \subset \sum_{(\theta, \omega)}=\{\lambda \in C: \lambda \neq \omega,|\arg (\lambda-\omega)|<\theta\}$,

(ii) $\|R(\lambda, A)\|_{L(X)} \leq M /|\lambda-\omega|, \lambda \in \sum_{(\theta, \omega)}$.

Definition 3 (see [11]). Let $A$ be a linear closed operator with domain $D(A)$ defined on $X$. One can call $A$ the generator of a solution operator if there exist $\omega \geq 0$ and strongly continuous functions $S_{q}: \mathbb{R}^{+} \rightarrow L(x)$ such that $\left\{\lambda^{q}: \operatorname{Re} \lambda>\omega\right\} \subset \rho(A)$ and

$$
\begin{array}{r}
\lambda^{q-1}\left(\lambda^{q} I-A\right)^{-1} x= \\
\int_{0}^{\infty} e^{-\lambda t} S_{q}(t) x d t \\
\operatorname{Re} \lambda>\omega, \quad x \in X .
\end{array}
$$

In this case, $S_{q}$ is called the solution operator generated by $A$.

Consider the space

$$
\begin{gathered}
\mathscr{B}_{b}=\left\{x:(-\infty, b] \longrightarrow X \text { such that }\left.x\right|_{J_{k}} \in C\left(J_{k}, X\right)\right. \\
\text { and there exist } x\left(t_{k}^{-}\right) \text {and } x\left(t_{k}^{+}\right) \\
\text {with } x\left(t_{k}\right)=x\left(t_{k}^{-}\right), x_{0}=\phi \in \mathscr{B}_{h}, \\
k=0,1,2, \ldots, m\},
\end{gathered}
$$

where $\left.x\right|_{J_{k}}$ is the restriction of $x$ to $J_{k}=\left(t_{k}, t_{k+1}\right], k=$ $0,1,2 \ldots, m$. Let $\|\cdot\|_{\mathscr{B}_{b}}$ be a seminorm in $\mathscr{B}_{b}$ defined by

$$
\|x\|_{\mathscr{B}_{b}}=\sup _{s \in J}\|x(s)\|+\|\phi\|_{\mathscr{B}_{h}}, \quad x \in \mathscr{B}_{b} .
$$

Lemma 4 (see [14]). If the functions $g: J \times \mathscr{B}_{h} \rightarrow X$, $f: J \times \mathscr{B}_{h} \times X \rightarrow X$ satisfy the uniform Hölder condition with the exponent $\beta \in(0,1]$ and $A$ is a sectorial operator, then a piecewise continuously differentiable function $x \in \mathscr{B}_{b}^{0}$ is a mild solution of

$$
\begin{gathered}
D_{t}^{q}\left[x(t)+g\left(t, x_{t}\right)\right]=A\left[x(t)+g\left(t, x_{t}\right)\right] \\
+J_{t}^{1-q} f\left(t, x_{t}, H x(t)\right), \\
t \in J=[0, b], \quad t \neq t_{k}, \\
\Delta x\left(t_{k}\right)=I_{k}\left(x\left(t_{k}^{-}\right)\right), \quad k=1,2, \ldots, m, \\
x_{0}=\phi \in \mathscr{B}_{h},
\end{gathered}
$$

if $x$ is a solution of the following fractional integral equation:

$x(t)$

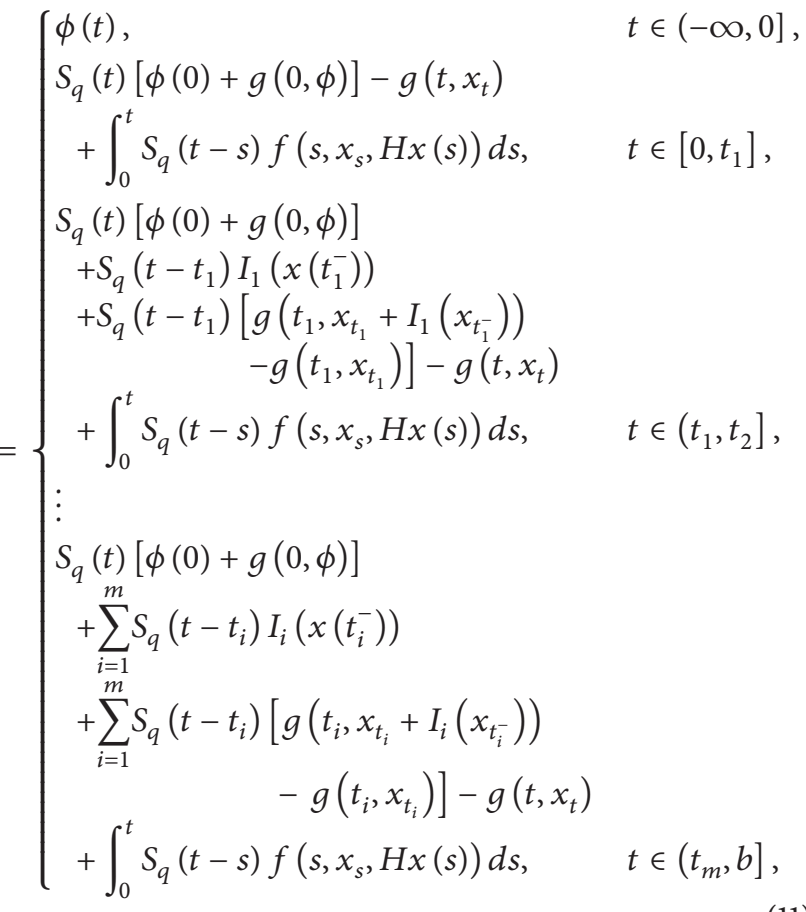

where $S_{q}(t)$ is the solution operator generated by A given by

$$
S_{q}(t)=E_{q, 1}\left(A t^{q}\right)=\frac{1}{2 \pi i} \int_{\widehat{B}_{r}} e^{\lambda t} \frac{\lambda^{q-1}}{\lambda^{q}-A} d \lambda,
$$

where $\widehat{B}_{r}$ denotes the Bromwich path.

Let $x_{b}(\phi ; u)$ be the state value of system (1) at terminal time $b$ corresponding to the control $u$ and the initial value $\phi \in \mathscr{B}_{h}$. Introduce the set $\mathscr{R}(b, \phi)=\left\{x_{b}(\phi ; u)(0): u(\cdot) \in\right.$ $\left.L^{2}(J, U)\right\}$, which is called the reachable set of system (1) at terminal time $b$.

Definition 5. The fractional control system (1) is said to be exactly controllable on the interval $J$ if $\mathscr{R}(b, \phi)=X$.

Assume that the linear fractional differential control system

$$
\begin{gathered}
D_{t}^{q} x(t)=A x(t)+(B u)(t), \quad t \in[0, b], \\
x(0)=\phi(0)
\end{gathered}
$$


is exactly controllable. It is convenient at this point to introduce the controllability operator associated with (13) as

$$
\Gamma_{0}^{b}=\int_{0}^{b} S_{q}(b-s) B B^{*} S_{q}^{*}(b-s) d s,
$$

where $B^{*}$ denotes the adjoint of $B$, and $S_{q}^{*}(t)$ is the adjoint of $S_{q}(t)$. It is straightforward that the operator $\Gamma_{0}^{b}$ is a linear bounded operator [19].

Lemma 6. If the linear fractional system (13) is exactly controllable if and only then for some $\gamma>0$ such that $\left\langle\Gamma_{0}^{b} x, x\right\rangle \geq$ $\gamma\|x\|^{2}$, for all $x \in X$ and consequently $\left\|\left(\Gamma_{0}^{b}\right)^{-1}\right\| \leq 1 / \gamma$.

In order to define the concept of mild solution for the control problem (1), by comparison with the impulsive neutral fractional equations given in [14], we associate problem (1) to the integral equation

$$
\begin{aligned}
& x(t) \\
& \begin{cases}\phi(t), & t \in(-\infty, 0] \\
S_{q}(t)[\phi(0)+g(0, \phi)]-g\left(t, x_{t}\right) & \\
+\int_{0}^{t} S_{q}(t-s) B u(s) d s & t \in\left[0, t_{1}\right] \\
+\int_{0}^{t} S_{q}(t-s) f\left(s, x_{s}, H x(s)\right) d s, & \\
S_{q}(t)[\phi(0)+g(0, \phi)] & \\
+S_{q}\left(t-t_{1}\right) I_{1}\left(x\left(t_{1}^{-}\right)\right) \\
+S_{q}\left(t-t_{1}\right)\left[g\left(t_{1}, x_{t_{1}}+I_{1}\left(x_{t_{1}^{-}}\right)\right)\right. \\
\left.\quad-g\left(t_{1}, x_{t_{1}}\right)\right]-g\left(t, x_{t}\right) \\
+\int_{0}^{t} S_{q}(t-s) B u(s) d s \\
+\int_{0}^{t} S_{q}(t-s) f\left(s, x_{s}, H x(s)\right) d s, \\
\vdots \\
+\int_{0}^{t} S_{q}(t-s) B u(s) d s \\
+\int_{0}^{t} S_{q}(t-s) f\left(s, x_{s}, H x(s)\right) d s, \\
S_{q}(t)[\phi(0)+g(0, \phi)] \\
+\sum_{i=1}^{m} S_{q}\left(t-t_{i}\right) I_{i}\left(x\left(t_{i}^{-}\right)\right) \\
+\sum_{i=1}^{m} S_{q}\left(t-t_{i}\right)\left[g\left(t_{i}, x_{t_{i}}+I_{i}\left(x_{t_{i}^{-}}\right)\right)\right.\end{cases}
\end{aligned}
$$

Definition 7. A function $x:(-\infty, b] \rightarrow X$ is said to be a mild solution for the system (1) if for each $u \in L^{2}(J, U)$, $x_{0}=\phi \in \mathscr{B}_{h}$ on $(-\infty, 0] ;\left.\Delta x\right|_{t=t_{k}}=I_{k}\left(x\left(t_{k}^{-}\right)\right), k=1, \ldots, m$, the restriction of $x(\cdot)$ to the interval $[0, b) \backslash\left\{t_{1}, \ldots, t_{m}\right\}$ is continuous, and the integral equation (15) is satisfied.

\section{Controllability Results}

In this section, we formulate and prove a set of sufficient conditions for the exact controllability of impulsive neutral fractional control differential system (1) by using the solution operator theory, fractional calculations, and fixed point argument. To prove the controllability result, we need the following hypotheses:

(H1) There exists a constant $M>0$ such that

$$
\left\|S_{q}(t)\right\|_{L(X)} \leq M, \quad \forall t \in[0, b]
$$

(H2) The function $g: J \times \mathscr{B}_{h} \rightarrow X$ is continuous, and there exists a constant $L_{g}>0$ such that

$$
\begin{array}{r}
\left\|g\left(t_{1}, \psi_{1}\right)-g\left(t_{2}, \psi_{2}\right)\right\|_{X} \leq L_{g}\left(\left|t_{1}-t_{2}\right|+\left\|\psi_{1}-\psi_{2}\right\|_{\mathscr{B}_{h}}\right), \\
t_{i} \in J, \psi_{i} \in \mathscr{B}_{h}, i=1,2 .
\end{array}
$$

(H3) There exist constants $\mu_{1}>0$ and $\mu_{2}>0$ such that

$$
\begin{gathered}
\|f(t, \varphi, x)-f(t, \psi, y)\|_{X} \leq \mu_{1}\|\varphi-\psi\|_{\mathscr{B}_{h}}+\mu_{2}\|x-y\|_{X}, \\
t \in J, \varphi, \psi \in \mathscr{B}_{h}, x, y \in X .
\end{gathered}
$$

(H4) $I_{k} \in C(X, X)$, and there exist constants $\rho>0$ such that

$$
\begin{array}{r}
\left\|I_{k}(x)-I_{k}(y)\right\|_{X} \leq \rho\|x-y\|_{X}, \quad x, y \in X \\
\text { for each } k=1, \ldots, m .
\end{array}
$$

(H5) The linear fractional system (13) is exactly controllable.

Theorem 8. Assume that the hypotheses (H1)-(H5) are satisfied, then the fractional impulsive system (1) is exactly controllable on J provided that

$$
\begin{aligned}
& \widehat{L}=\left(1+\frac{1}{\gamma} M_{B}^{2} M^{2}\right)[ m M \rho+m M L_{g} C_{1}^{*}(2+\rho) \\
&\left.+L_{g} C_{1}^{*}+M b\left(\mu_{1} C_{1}^{*}+\mu_{2} H^{*}\right)\right]<1,
\end{aligned}
$$

where $C_{1}^{*}=\sup _{0<\tau<b} C_{1}(\tau)$ and $H^{*}=\sup _{t \in[0, b]} \int_{0}^{t} G(t, s) d s<$ $\infty$.

Proof. For an arbitrary function $x(\cdot)$, choose the feedback control function as follows: 


$$
u_{x}(t)= \begin{cases}B^{*} S_{q}^{*}\left(t_{1}-t\right)\left(\Gamma_{0}^{t_{1}}\right)^{-1} & \\ & \times\left[x_{t_{1}}-S_{q}\left(t_{1}\right)[\phi(0)+g(0, \phi)]+g\left(t_{1}, x_{t_{1}}\right)-\int_{0}^{t_{1}} S_{q}\left(t_{1}-s\right) \times f\left(s, x_{s}, H x(s)\right) d s\right], \\ & B^{*} S_{q}^{*}\left(t_{2}-t\right)\left(\Gamma_{0}^{t_{2}}\right)^{-1} \\ & \times\left[x_{t_{1}}-S_{q}\left(t_{2}\right)[\phi(0)+g(0, \phi)]-S_{q}\left(t_{2}-t_{1}\right) I_{1}\left(x\left(t_{1}^{-}\right)\right)\right. \\ - & \left.S_{q}\left(t_{2}-t_{1}\right)\left[g\left(t_{1}, x_{t_{1}}+I_{1}\left(x_{t_{1}^{-}}\right)\right)-g\left(t_{1}, x_{t_{1}}\right)\right]+g\left(t_{2}, x_{t_{2}}\right)-\int_{0}^{t_{2}} S_{q}\left(t_{2}-s\right) \times f\left(s, x_{s}, H x(s)\right) d s\right], t \in\left(t_{1}, t_{2}\right], \\ \vdots & \\ B^{*} S_{q}^{*}(b-t)\left(\Gamma_{0}^{b}\right)^{-1} & \\ & \times\left[x_{b}-S_{q}(b)[\phi(0)+g(0, \phi)]-\sum_{i=1}^{m} S_{q}\left(b-t_{i}\right) I_{i}\left(x\left(t_{i}^{-}\right)\right)\right. \\ & -\sum_{i=1}^{m} S_{q}\left(b-t_{i}\right)\left[g\left(t_{i}, x_{t_{i}}+I_{i}\left(x_{t_{i}^{-}}\right)\right)-g\left(t_{i}, x_{t_{i}}\right)\right]+g\left(b, x_{b}\right) \\ \left.-\int_{0}^{b} S_{q}(b-s) f\left(s, x_{s}, H x(s)\right) d s\right], & t \in\left(t_{m}, b\right]\end{cases}
$$

and define the operator $\Phi: \mathscr{B}_{b} \rightarrow \mathscr{B}_{b}$ by

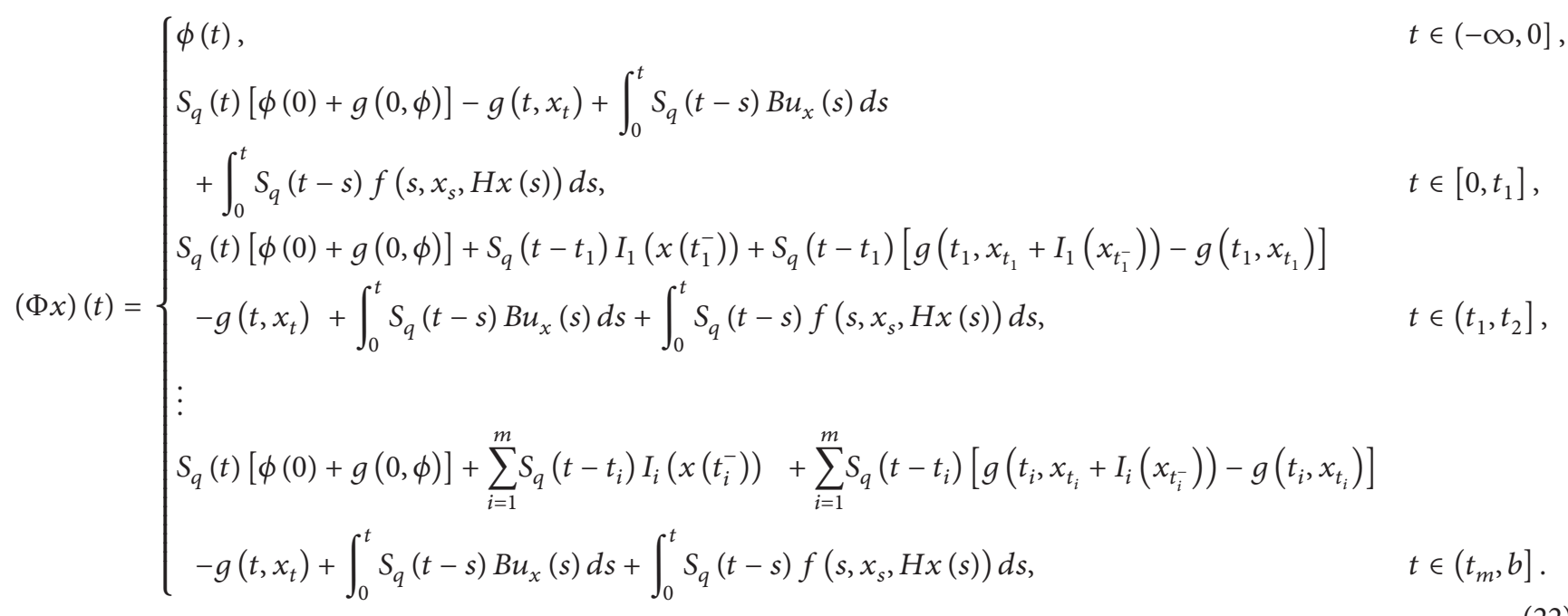

It should be noted that the control (21) transfers the system (1) from the initial state $\phi$ to the final state $x_{b}$ provided that the operator $\Phi$ has a fixed point. In order to prove the exact controllability result, it is enough to show that the operator $\Phi$ has a fixed point in $\mathscr{B}_{b}$.

Define the function $y(\cdot):(-\infty, b] \rightarrow X$ by

$$
y(t)= \begin{cases}\phi(t), & t \in(-\infty, 0], \\ 0, & t \in[0, b],\end{cases}
$$

then $y_{0}=\phi$. For each $z \in C(J, R)$ with $z(0)=0$, let the function $\bar{z}$ be defined by

$$
\bar{z}(t)= \begin{cases}0, & t \in(-\infty, 0], \\ z(t), & t \in[0, b] .\end{cases}
$$

If $x(\cdot)$ satisfies (15), then we can decompose $x(\cdot)$ as $x(t)=$ $y(t)+\bar{z}(t)$ for $t \in J$, which implies that $x_{t}=y_{t}+\bar{z}_{t}$ for $t \in J$ and the function $z(\cdot)$ satisfies 


$$
z(t)= \begin{cases}S_{q}(t)[\phi(0)+g(0, \phi)]-g\left(t, y_{t}+\bar{z}_{t}\right) \\ \quad+\int_{0}^{t} S_{q}(t-s) B u_{y+\bar{z}}(s) d s+\int_{0}^{t} S_{q}(t-s) \times f\left(s, y_{s}+\bar{z}_{s}, H(y(s)+\bar{z}(s))\right) d s, \quad t \in\left[0, t_{1}\right] \\ S_{q}(t)[\phi(0)+g(0, \phi)]+S_{q}\left(t-t_{1}\right) I_{1}\left(y\left(t_{1}^{-}\right)+\bar{z}\left(t_{1}^{-}\right)\right)+S_{q}\left(t-t_{1}\right) \\ \quad \times\left[g\left(t_{1}, y_{t_{1}}+\bar{z}_{t_{1}}+I_{1}\left(y_{t_{1}^{-}}+\bar{z}_{t_{1}^{-}}\right)\right)-g\left(t_{1}, y_{t_{1}}+\bar{z}_{t_{1}}\right)\right]-g\left(t, y_{t}+\bar{z}_{t}\right) \\ \quad+\int_{0}^{t} S_{q}(t-s) B u_{y+\bar{z}}(s) d s+\int_{0}^{t} S_{q}(t-s) f\left(s, y_{s}+\bar{z}_{s}, H(y(s)+\bar{z}(s))\right) d s, & t \in\left(t_{1}, t_{2}\right] \\ \vdots & \\ & \\ & \\ & (t)[\phi(0)+g(0, \phi)]+\sum_{i=1}^{m} S_{q}\left(t-t_{i}\right) I_{i}\left(y\left(t_{i}^{-}\right)+\bar{z}\left(t_{i}^{-}\right)\right) \\ & +\sum_{i=1}^{m} S_{q}\left(t-t_{i}\right)\left[g\left(t_{i}, y_{t_{i}}+\bar{z}_{t_{i}}+I_{i}\left(y_{t_{i}^{-}}+\bar{z}_{t_{i}^{-}}\right)\right)-g\left(t_{i}, y_{t_{i}}+\bar{z}_{t_{i}}\right)-g\left(t, y_{t}+\bar{z}_{t}\right)\right]\end{cases}
$$

where

$$
\begin{aligned}
& u_{y+\bar{z}}(t) \\
& =\left\{\begin{array}{l}
B^{*} S_{q}^{*}\left(t_{1}-t\right)\left(\Gamma_{0}^{t_{1}}\right)^{-1} \\
\times\left[x_{t_{1}}-S_{q}\left(t_{1}\right)[\phi(0)+g(0, \phi)]+g\left(t_{1}, y_{t_{1}}+\bar{z}_{t_{1}}\right)-\int_{0}^{t_{1}} S_{q}\left(t_{1}-s\right) f\left(s, y_{s}+\bar{z}_{s}, H(y(s)+\bar{z}(s)) d s\right)\right], \quad t \in\left[0, t_{1}\right], \\
B^{*} S_{q}^{*}\left(t_{2}-t\right)\left(\Gamma_{0}^{t_{2}}\right)^{-1} \times\left[x_{t_{1}}-S_{q}\left(t_{2}\right)[\phi(0)+g(0, \phi)]-S_{q}\left(t_{2}-t_{1}\right) I_{1}\left(y\left(t_{1}^{-}\right)+\bar{z}\left(t_{1}^{-}\right)\right)\right. \\
\quad-S_{q}\left(t_{2}-t_{1}\right)\left[g\left(t_{1}, y_{t_{1}}+\bar{z}_{t_{1}}+I_{1}\left(y_{t_{1}^{-}}+\bar{z}_{t_{1}^{-}}\right)\right)-g\left(t_{1}, y_{t_{1}}+\bar{z}_{t_{1}}\right)\right] \\
\left.\quad+g\left(t_{2}, y_{t_{2}}+\bar{z}_{t_{2}}\right)-\int_{0}^{t_{2}} S_{q}\left(t_{2}-s\right) \times f\left(s, y_{s}+\bar{z}_{s}, H(y(s)+\bar{z}(s))\right) d s\right], \\
\vdots \quad B^{*} S_{q}^{*}(b-t)\left(\Gamma_{0}^{b}\right)^{-1} \times\left[x_{b}-S_{q}(b)[\phi(0)+g(0, \phi)]-\sum_{i=1}^{m} S_{q}\left(b-t_{i}\right) I_{i}\left(y\left(t_{i}^{-}\right)+\bar{z}\left(t_{i}^{-}\right)\right)\right. \\
\quad-\sum_{i=1}^{m} S_{q}\left(b-t_{i}\right) g\left(t_{i}, y_{t_{i}}+\bar{z}_{t_{i}}+I_{i}\left(y_{t_{i}^{-}}+\bar{z}_{t_{i}^{-}}\right)\right)-g\left(t_{i}, y_{t_{i}}+\bar{z}_{t_{i}}\right)+g\left(b, y_{b}+\bar{z}_{b}\right) \\
\left.-\int_{0}^{b} S_{q}(b-s) \times f\left(s, y_{s}+\bar{z}_{s}, H(y(s)+\bar{z}(s))\right) d s\right], \\
\quad t \in\left(t_{1}, t_{2}\right]
\end{array},\right.
\end{aligned}
$$

Let $\mathscr{B}_{b}^{0}=\left\{z \in \mathscr{B}_{b}: z_{0}=0 \in \mathscr{B}_{h}\right\}$. For any $z \in \mathscr{B}_{b}^{0}$, we get $\|z\|_{\mathscr{B}_{b}^{0}}=\sup _{s \in J}\|z(s)\|_{X}+\left\|z_{0}\right\|_{\mathscr{B}_{h}}=\sup _{s \in J}\|z(s)\|_{X}, \quad z \in \mathscr{B}_{b}^{0}$.
Thus, $\left(\mathscr{B}_{b}^{0},\|\cdot\|_{\mathscr{B}_{b}^{0}}\right)$ is a Banach space. Define the operator $\Pi$ : $\mathscr{B}_{b}^{0} \rightarrow \mathscr{B}_{b}^{0}$ by 


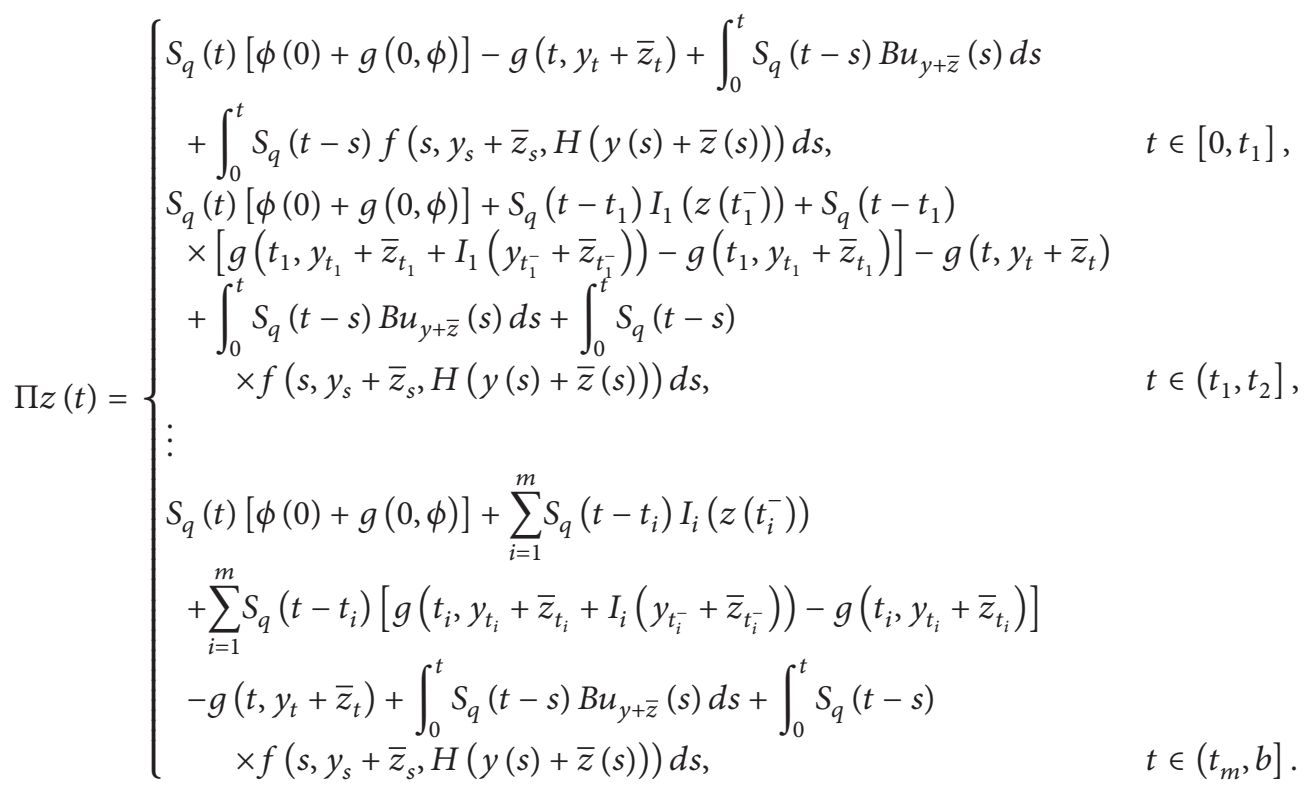

It can be easily seen that the operator $\Phi$ has a fixed point if and only if $\Pi$ has a fixed point. Now, we will prove that $\Pi$ has a unique fixed point. In order to prove this, we show that $\Pi$ is a contraction mapping. Let $z, z^{*} \in \mathscr{B}_{b}^{0}$, for all $t \in\left[0, t_{1}\right]$, we have

$$
\begin{aligned}
& \left\|(\Pi z)(t)-\left(\Pi z^{*}\right)(t)\right\|_{X} \\
& \leq\left\|g\left(t, y_{t}+\bar{z}_{t}\right)-g\left(t, y_{t}+\bar{z}_{t}^{*}\right)\right\|_{X} \\
& +\int_{0}^{t}\left\|S_{q}(t-\eta) B B^{*} S_{q}^{*}\left(t_{1}-\eta\right)\left(\Gamma_{0}^{t_{1}}\right)^{-1}\right\|_{X} \\
& \times\left[\left\|g\left(t_{1}, y_{t_{1}}+\bar{z}_{t_{1}}\right)-g\left(t_{1}, y_{t_{1}}+\bar{z}_{t_{1}}^{*}\right)\right\|_{X}\right. \\
& +\int_{0}^{t_{1}}\left\|S_{q}\left(t_{1}-s\right)\right\|_{L(X)} \\
& \times \| f\left(s, y_{s}+\bar{z}_{s}, H(y(s)+\bar{z}(s))\right) \\
& -f\left(s, y_{s}+\bar{z}_{s}^{*},\right. \\
& \left.\left.H\left(y(s)+\bar{z}^{*}(s)\right)\right) \|_{X} d s\right] d \eta \\
& +\int_{0}^{t}\left\|S_{q}(t-s)\right\|_{L(X)} \\
& \times \| f\left(s, y_{s}+\bar{z}_{s}, H(y(s)+\bar{z}(s))\right) \\
& -f\left(s, y_{s}+\bar{z}_{s}^{*}, H\left(y(s)+\bar{z}^{*}(s)\right)\right) \|_{X} d s \\
& \leq L_{g}\left\|\bar{z}_{t}-\bar{z}_{t}^{*}\right\|_{\mathscr{B}_{h}} \\
& +\frac{1}{\gamma} M_{B}^{2} M^{2} \int_{0}^{t}\left[L_{g}\left\|\bar{z}_{t}-\bar{z}_{t}^{*}\right\|_{\mathscr{B}_{h}}\right.
\end{aligned}
$$

$$
\begin{gathered}
+M \int_{0}^{t_{1}}\left(\mu_{1}\left\|\bar{z}_{s}-\bar{z}_{s}^{*}\right\|_{\mathscr{B}_{h}}+\mu_{2}\right. \\
\times \| H(y(s)-\bar{z}(s)) \\
-H(y(s)
\end{gathered}
$$$$
\left.\left.\left.-\bar{z}^{*}(s)\right) \|_{X}\right) d s\right] d \eta
$$$$
+M \int_{0}^{t_{1}}\left(\mu_{1}\left\|\bar{z}_{s}-\bar{z}_{s}^{*}\right\|_{\mathscr{B}_{h}}+\mu_{2} \| H(y(s)-\bar{z}(s))\right.
$$$$
\left.-H\left(y(s)-\bar{z}^{*}(s)\right) \|_{X}\right) d s
$$$$
\leq\left(1+\frac{1}{\gamma} M_{B}^{2} M^{2}\right)\left[L_{g} C_{1}^{*}+M b\left(\mu_{1} C_{1}^{*}+\mu_{2} H^{*}\right)\right]
$$$$
\times\left\|z-z^{*}\right\|_{\mathscr{B}_{b}^{0}} .
$$

Similarly, for $t \in\left(t_{k}, t_{k+1}\right], k=1,2, \ldots, m$, we can obtain $\left\|(\Pi z)(t)-\left(\Pi z^{*}\right)(t)\right\|_{X}$

$$
\begin{gathered}
\leq \sum_{i=1}^{k}\left\|S_{q}\left(t-t_{i}\right)\right\|_{L(x)}\left\|I_{i}\left(z\left(t_{i}^{-}\right)\right)-I_{i}\left(z\left(t_{i}^{-}\right)\right)\right\|_{X} \\
+\sum_{i=1}^{k}\left\|S_{q}\left(t-t_{i}\right)\right\|_{L(X)} \\
\times\left[\| g\left(t_{i}, y_{t_{i}}+\bar{z}_{t_{i}}+I_{i}\left(y_{t_{i}^{-}}+\bar{z}_{t_{i}^{-}}\right)\right)\right. \\
-g\left(t_{i}, y_{t_{i}}+\bar{z}_{t_{i}}^{*}+I_{i}\left(y_{t_{i}^{-}}+\bar{z}_{t_{i}^{-}}^{*}\right)\right) \|_{X}
\end{gathered}
$$




$$
\begin{aligned}
& \left.+\left\|g\left(t_{i}, y_{t_{i}}+\bar{z}_{t_{i}}\right)-g\left(t_{i}, y_{t_{i}}+\bar{z}_{t_{i}}^{*}\right)\right\|_{X}\right] \\
& +\left\|g\left(t, y_{t}+\bar{z}_{t}\right)-g\left(t, y_{t}+\bar{z}_{t}^{*}\right)\right\|_{X} \\
& +\int_{0}^{t}\left\|S_{q}(t-\eta) B B^{*} S_{q}^{*}\left(t_{k+1}-\eta\right)\left(\Gamma_{0}^{t_{1}}\right)^{-1}\right\|_{X} \\
& \times\left[\sum_{i=1}^{k}\left\|S_{q}\left(t_{k+1}-t_{i}\right)\right\|_{L(x)}\left\|I_{i}\left(z\left(t_{i}^{-}\right)\right)-I_{i}\left(z\left(t_{i}^{-}\right)\right)\right\|_{X}\right. \\
& +\sum_{i=1}^{k}\left\|S_{q}\left(t_{k+1}-t_{i}\right)\right\|_{L(X)} \\
& \times\left[\| g\left(t_{i}, y_{t_{i}}+\bar{z}_{t_{i}}+I_{i}\left(y_{t_{i}^{-}}+\bar{z}_{t_{i}^{-}}\right)\right)\right. \\
& -g\left(t_{i}, y_{t_{i}}+\bar{z}_{t_{i}}^{*}+I_{i}\left(y_{t_{i}^{-}}+\bar{z}_{t_{i}^{-}}^{*}\right)\right) \|_{X} \\
& \left.+\left\|g\left(t_{i}, y_{t_{i}}+\bar{z}_{t_{i}}\right)-g\left(t_{i}, y_{t_{i}}+\bar{z}_{t_{i}}^{*}\right)\right\|_{X}\right] \\
& +\left\|g\left(t_{k+1}, y_{t_{k+1}}+\bar{z}_{t_{k+1}}\right)-g\left(t_{k+1}, y_{t_{k+1}}+\bar{z}_{t_{k+1}}^{*}\right)\right\|_{X} \\
& +\int_{0}^{t_{k+1}}\left\|S_{q}\left(t_{k+1}-s\right)\right\|_{L(X)} \\
& \times \| f\left(s, y_{s}+\bar{z}_{s}, H(y(s)+\bar{z}(s))\right) \\
& \left.-f\left(s, y_{s}+\bar{z}_{s}^{*}, H\left(y(s)+\bar{z}^{*}(s)\right)\right) \|_{X} d s\right] d \eta \\
& +\int_{0}^{t}\left\|S_{q}(t-s)\right\|_{L(X)} \\
& \times \| f\left(s, y_{s}+\bar{z}_{s}, H(y(s)+\bar{z}(s))\right) \\
& -f\left(s, y_{s}+\bar{z}_{s}^{*}, H\left(y(s)+\bar{z}^{*}(s)\right)\right) \|_{X} d s \\
& \leq\left(1+\frac{1}{\gamma} M_{B}^{2} M^{2}\right)\left[k M \rho+k M L_{g} C_{1}^{*}(2+\rho)+L_{g} C_{1}^{*}\right. \\
& \left.+M b\left(\mu_{1} C_{1}^{*}+\mu_{2} H^{*}\right)\right]\left\|z-z^{*}\right\|_{\mathscr{B}_{b}^{0}} .
\end{aligned}
$$

Thus, for all $t \in[0, b]$, we get the estimate

$$
\begin{aligned}
& \left\|(\Pi z)(t)-\left(\Pi z^{*}\right)(t)\right\|_{X} \\
& \leq\left(1+\frac{1}{\gamma} M_{B}^{2} M^{2}\right)\left[m M \rho+m M L_{g} C_{1}^{*}(2+\rho)+L_{g} C_{1}^{*}\right. \\
& \left.\quad+M b\left(\mu_{1} C_{1}^{*}+\mu_{2} H^{*}\right)\right]\left\|z-z^{*}\right\|_{\mathscr{B}_{b}^{0}} \\
& \leq \hat{L}\left\|z-z^{*}\right\|_{\mathscr{B}_{b}^{0}}
\end{aligned}
$$

Thus, we have $\left\|(\Pi z)(t)-\left(\Pi z^{*}\right)(t)\right\|_{X} \leq \widehat{L}\left\|z-z^{*}\right\|_{\mathscr{B}_{b}^{0}}$ for all $t \in[0, b]$. Since $\widehat{L}<1$, this implies that $\Pi$ is a contraction mapping, and hence, $\Pi$ has a unique fixed point $z \in \mathscr{B}_{b}^{0}$. Thus, the system (1) is exactly controllable on $[0, b]$. The proof is complete.

However, the concept of exact controllability is very limited for many dynamic control systems, and the approximate controllability is more appropriate for these control systems instead of exact controllability. Taking this into account, in this paper, we will also discuss the approximate controllability result of the nonlinear impulsive fractional control system (1). The control system is said to be approximately controllable if, for every initial data $\phi$ and every finite time horizon $b>0$, an admissible control process can be found such that the corresponding solution is arbitrarily close to a given square integrable final condition. Further, approximate controllable systems are more prevalent, and often, approximate controllability is completely adequate in applications. In recent years, for deterministic and stochastic control systems including delay term, there are several papers devoted to the study of approximate controllability [23-25]. Sukavanam and Kumar [26] obtained a set of conditions which ensure the approximate controllability of a class of semilinear fractional delay control systems. Recently, Sakthivel et al. [23] formulated and proved a new set of sufficient conditions for approximate controllability of fractional differential equations by using the fractional calculus theory and solutions operators.

Definition 9. The fractional control system (1) is said to be approximately controllable on $[0, b]$ if the closure of the reachable set $\overline{\mathscr{R}(b, \phi)}$ is dense in $X$; that is, $\overline{\mathscr{R}(b, \phi)}=X$.

Remark 10. Assume that the linear fractional control system

$$
\begin{gathered}
D_{t}^{q} x(t)=A x(t)+(B u)(t), \quad t \in[0, b], \\
x(0)=\phi
\end{gathered}
$$

is approximate controllable. Let us now introduce the operators associated with (32) as $\Gamma_{0}^{b}=\int_{0}^{b} \mathcal{S}_{q}(b-s) B B^{*} \mathcal{S}_{q}^{*}(b-$ s)ds, $R\left(\alpha, \Gamma_{0}^{b}\right)=\left(\alpha I+\Gamma_{0}^{b}\right)^{-1}$ for $\alpha>0$. It should be mentioned that the approximate controllability of (32) is equivalent to the convergence of function $\alpha R\left(\alpha, \Gamma_{0}^{b}\right)$ to zero, as $\alpha \rightarrow 0^{+}$ in the strong operator topology (see $[23,27]$ and references therein).

Theorem 11. Assume that conditions (H1)-(H4) hold and that the family $\left\{S_{q}(t): t>0\right\}$ is compact. In addition, assume that the function $f$ is uniformly bounded and the linear system associated with the system (1) is approximately controllable, then the nonlinear fractional control system with infinite delay (1) is approximately controllable on $[0, b]$.

Proof. For each $\alpha>0$, define the operator $\Psi: \mathscr{B}_{b} \rightarrow \mathscr{B}_{b}$ by $\Psi x(t)=z(t)$, where 


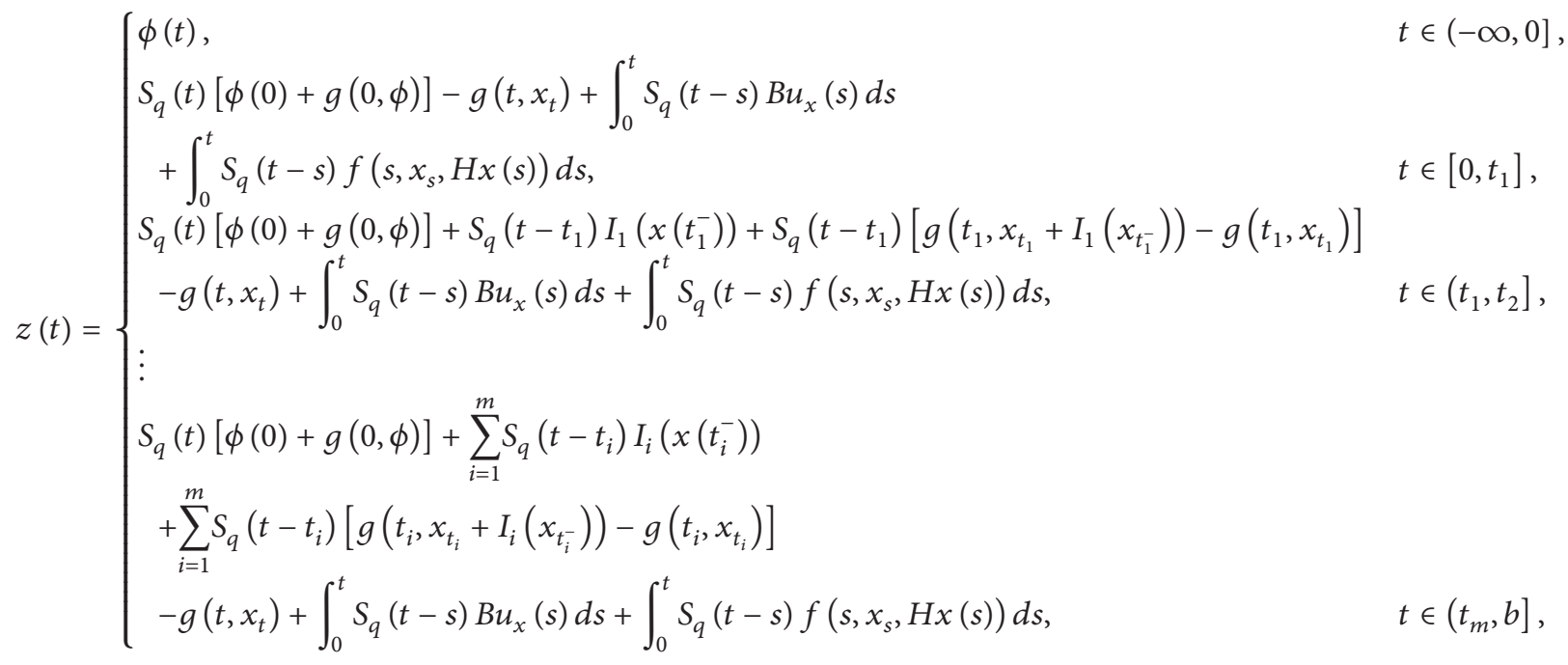

$$
\begin{aligned}
& u_{x}(t)= \begin{cases}B^{*} S_{q}^{*}\left(t_{1}-t\right) R\left(\alpha, \Gamma_{0}^{t_{1}}\right) p(x(\cdot)), & t \in\left[0, t_{1}\right], \\
B^{*} S_{q}^{*}\left(t_{2}-t\right) R\left(\alpha, \Gamma_{t_{1}}^{t_{2}}\right) p(x(\cdot)), & t \in\left(t_{1}, t_{2}\right], \\
\vdots & \\
B^{*} S_{q}^{*}(b-t) R\left(\alpha, \Gamma_{t_{m}}^{b}\right) p(x(\cdot)), & t \in\left(t_{m}, b\right],\end{cases}
\end{aligned}
$$

where

$p(x(\cdot))$

$$
\left\{\begin{array}{c}
x_{t_{1}}-S_{q}\left(t_{1}\right)[\phi(0)+g(0, \phi)]+g\left(t_{1}, x_{t_{1}}\right) \\
-\int_{0}^{t_{1}} S_{q}\left(t_{1}-s\right) f\left(s, x_{s}, H x(s)\right) d s, \\
x_{t_{1}}-S_{q}\left(t_{2}\right)[\phi(0)+g(0, \phi)] \\
-S_{q}\left(t_{2}-t_{1}\right) I_{1}\left(x\left(t_{1}^{-}\right)\right) \\
-S_{q}\left(t_{2}-t_{1}\right)\left[g\left(t_{1}, x_{t_{1}}+I_{1}\left(x_{t_{1}^{-}}\right)\right)\right. \\
\left.-g\left(t_{1}, x_{t_{1}}\right)\right]+g\left(t_{2}, x_{t_{2}}\right) \\
-\int_{0}^{t_{2}} S_{q}\left(t_{2}-s\right) f\left(s, x_{s}, H x(s)\right) d s, \\
\vdots \begin{array}{l}
x_{b}-S_{q}(b)[\phi(0)+g(0, \phi)] \\
-\sum_{i=1}^{m} S_{q}\left(b-t_{i}\right) I_{i}\left(x\left(t_{i}^{-}\right)\right) \\
-\sum_{i=1}^{m} S_{q}\left(b-t_{i}\right)\left[g\left(t_{i}, x_{t_{i}}+I_{i}\left(x_{t_{i}^{-}}\right)\right)\right. \\
\left.-g\left(t_{i}, x_{t_{i}}\right)\right]+g\left(b, x_{b}\right) \\
-\int_{0}^{b} S_{q}(b-s) f\left(s, x_{s}, H x(s)\right) d s,
\end{array} \quad t \in\left(t_{m}, b\right] .
\end{array}\right.
$$

One can easily show that for all $\alpha>0$, the operator $\Psi$ has a fixed point by employing the technique used in Theorem 8 with some changes.
Let $\hat{x}^{\alpha}(\cdot)$ be a fixed point of $\Psi$. Further, any fixed point of $\Psi$ is a mild solution of (1) under the control

$$
\widehat{u}^{\alpha}(t)= \begin{cases}B^{*} S_{q}^{*}\left(t_{1}-t\right) R\left(\alpha, \Gamma_{0}^{t_{1}}\right) p\left(\widehat{x}^{\alpha}\right), & t \in\left[0, t_{1}\right], \\ B^{*} S_{q}^{*}\left(t_{2}-t\right) R\left(\alpha, \Gamma_{0}^{t_{2}}\right) p\left(\widehat{x}^{\alpha}\right), & t \in\left(t_{1}, t_{2}\right], \\ \vdots & \\ B^{*} S_{q}^{*}(b-t) R\left(\alpha, \Gamma_{0}^{b}\right) p\left(\widehat{x}^{\alpha}\right), & t \in\left(t_{m}, b\right]\end{cases}
$$

and satisfies

$$
\begin{array}{cc}
\widehat{x}^{\alpha}\left(t_{1}\right)=x_{t_{1}}-\alpha R\left(\alpha, \Gamma_{0}^{t_{1}}\right) p\left(\widehat{x}^{\alpha}\right), & t \in\left[0, t_{1}\right], \\
\widehat{x}^{\alpha}\left(t_{2}\right)=x_{t_{2}}-\alpha R\left(\alpha, \Gamma_{0}^{t_{2}}\right) p\left(\widehat{x}^{\alpha}\right), & t \in\left(t_{1}, t_{2}\right], \\
\vdots & \\
\widehat{x}^{\alpha}(b)=x_{b}-\alpha R\left(\alpha, \Gamma_{0}^{b}\right) p\left(\widehat{x}^{\alpha}\right), & t \in\left(t_{m}, b\right] .
\end{array}
$$

Moreover, by the assumption that $f$ is uniformly bounded, there exists $N>0$ such that

$$
\int_{0}^{b}\left\|f\left(s, \widehat{x}_{s}^{\alpha}, H \widehat{x}^{\alpha}(s)\right)\right\|^{2} d s \leq b N^{2},
$$

and consequently, the sequence $\left\{f\left(s, \widehat{x}_{s}^{\alpha}, H \widehat{x}^{\alpha}(s)\right)\right\}$ is bounded in $L^{2}(J, X)$. Then, there is a subsequence still denoted 
by $\left\{f\left(s, \widehat{x}_{s}^{\alpha}, H \widehat{x}^{\alpha}(s)\right)\right\}$ that converges weakly to say $f(s)$ in $L^{2}(J, X)$. Now, we define

$w$

$$
=\left\{\begin{array}{lr}
x_{t_{1}}-S_{q}\left(t_{1}\right)[\phi(0)+g(0, \phi)] & \\
& +g\left(t_{1}, x_{t_{1}}^{\alpha}\right)-\int_{0}^{t_{1}} S_{q}\left(t_{1}-s\right) f(s) d s, \\
x_{t_{1}}-S_{q}\left(t_{2}\right)[\phi(0)+g(0, \phi)] & t \in\left[0, t_{1}\right], \\
-S_{q}\left(t_{2}-t_{1}\right) I_{1}\left(x^{\alpha}\left(t_{1}^{-}\right)\right) & \\
-S_{q}\left(t_{2}-t_{1}\right)\left[g\left(t_{1}, x_{t_{1}}^{\alpha}+I_{1}\left(x_{t_{1}^{-}}^{\alpha}\right)\right)\right. & \\
\left.-g\left(t_{1}, x_{t_{1}}^{\alpha}\right)\right]+g\left(t_{2}, x_{t_{2}}^{\alpha}\right) & \\
-\int_{0}^{t_{2}} S_{q}\left(t_{2}-s\right) f(s) d s, & \\
\vdots & \\
x_{b}-S_{q}(b)[\phi(0)+g(0, \phi)] & \\
-\sum_{i=1}^{m} S_{q}\left(b-t_{i}\right) I_{i}\left(x^{\alpha}\left(t_{i}^{-}\right)\right) & \\
-\sum_{i=1}^{m} S_{q}\left(b-t_{i}\right)\left[g\left(t_{i}, x_{t_{i}}^{\alpha}+I_{i}\left(x_{t_{i}^{-}}^{\alpha}\right)\right)\right. & \\
\left.-g\left(t_{i}, x_{t_{i}}^{\alpha}\right)\right]+g\left(b, x_{b}^{\alpha}\right) & \\
-\int_{0}^{b} S_{q}(b-s) f(s) d s, & t \in\left(t_{m}, b\right] .
\end{array}\right.
$$

Now, for $t \in\left[0, t_{1}\right]$, we have

$$
\begin{aligned}
& \left\|p\left(\widehat{x}^{\alpha}\right)-w\right\| \\
& \quad=\left\|\int_{0}^{t_{1}} S_{q}\left(t_{1}-s\right)\left[f\left(s, \hat{x}_{s}^{\alpha}, H \widehat{x}^{\alpha}(s)\right)-f(s)\right] d s\right\| \\
& \quad \leq \sup _{t \in\left[0, t_{1}\right]}\left\|\int_{0}^{t} S_{q}(t-s)\left[f\left(s, \widehat{x}_{s}^{\alpha}, H \hat{x}^{\alpha}(s)\right)-f(s)\right] d s\right\| .
\end{aligned}
$$

Also, for $t \in\left[t_{i}, t_{i+1}\right], i=1, \ldots, m$, we have

$$
\begin{aligned}
& \left\|p\left(\widehat{x}^{\alpha}\right)-w\right\| \\
& \quad=\left\|\int_{0}^{t_{i+1}} S_{q}\left(t_{i+1}-s\right)\left[f\left(s, \widehat{x}_{s}^{\alpha}, H \widehat{x}^{\alpha}(s)\right)-f(s)\right] d s\right\| \\
& \quad \leq \sup _{t \in\left(t_{i}, t_{i+1}\right]}\left\|\int_{0}^{t} S_{q}(t-s)\left[f\left(s, \widehat{x}_{s}^{\alpha}, H \widehat{x}^{\alpha}(s)\right)-f(s)\right] d s\right\| .
\end{aligned}
$$

By using infinite-dimensional version of the Ascoli-Arzela theorem, it is easy to show that an operator $l(\cdot) \rightarrow \int_{0}^{\int} S_{q}(\cdot-$ $s) l(s) d s: L^{1}(J, X) \rightarrow C(J, X)$ is compact. Hence, for all $t \in[0, b]$, we obtain that $\left\|p\left(\hat{x}^{\alpha}\right)-w\right\| \rightarrow 0$ as $\alpha \rightarrow 0^{+}$. Moreover, from (36) we get for $t \in\left[0, t_{1}\right]$,

$$
\begin{aligned}
\left\|\widehat{x}^{\alpha}\left(t_{1}\right)-x_{t_{1}}\right\| \leq & \left\|\alpha R\left(\alpha, \Gamma_{0}^{t_{1}}\right)(w)\right\| \\
& +\left\|\alpha R\left(\alpha, \Gamma_{0}^{t_{1}}\right)\right\|\left\|p\left(\widehat{x}^{\alpha}\right)-w\right\| \\
\leq & \left\|\alpha R\left(\alpha, \Gamma_{0}^{t_{1}}\right)(w)\right\|+\left\|p\left(\widehat{x}^{\alpha}\right)-w\right\| .
\end{aligned}
$$

It follows from Remark 10 and (39) that $\left\|\widetilde{x}_{\alpha}\left(t_{1}\right)-x_{t_{1}}\right\| \rightarrow 0$ as $\alpha \rightarrow 0^{+}$. Similarly, in the view of (40), for $t \in\left(t_{i}, t_{i+1}\right]$, $i=1, \ldots, m$,

$$
\left\|\widehat{x}^{\alpha}\left(t_{i+1}\right)-x_{t_{i+1}}\right\| \longrightarrow 0 \quad \text { as } \alpha \longrightarrow 0^{+} .
$$

Thus, for all $t \in[0, b]$, we get $\left\|\widehat{x}^{\alpha}(b)-x_{b}\right\| \rightarrow 0$ as $\alpha \rightarrow 0^{+}$. This proves the approximate controllability of (1). The proof is completed.

Example 12. Now, we present an example to illustrate the abstract results of this paper which do not aim at generality but indicate how our theorem can be applied to concrete problems. Let $X=L^{2}[0, \pi]$. Define $A: X \rightarrow X$ by $A z=z^{\prime \prime}$ with domain $D(A)=\{z \in X$ : $z, z^{\prime}$ are absolutely continuous, $\left.z^{\prime \prime} \in X, z(0)=z(\pi)=0\right\}$. Then, $A$ generates an analytic semigroup $\{T(t), t>0\}$ in $X$, and it is given by [14]

$$
T(t) z=\sum_{n=1}^{\infty} e^{-n^{2} t}\left(z, e_{n}\right) e_{n}, \quad z \in X,
$$

where $e_{n}(y)=\sqrt{2 / \pi} \sin n y, n=1,2,3, \ldots$ is the orthogonal set of eigenvectors of $A$. Also, define an infinite dimensional control space $U$ by $U=\left\{u \mid u=\sum_{n=2}^{\infty} u_{n} e_{n}\right.$ with $\sum_{n=2}^{\infty} u_{n}^{2}<$ $\infty\}$ with norm defined by $\|u\|_{U}=\left(\sum_{n=2}^{\infty} u_{n}^{2}\right)^{1 / 2}$. Define a continuous linear map $B$ from $U$ to $X$ as $B u=2 u_{2} e_{1}+$ $\sum_{n=2}^{\infty} u_{n} e_{n}$ for $u=\sum_{n=2}^{\infty} u_{n} e_{n} \in U$.

Note that the subordination principle of solution operator implies that $A$ is the infinitesimal generator of a solution operator $\left\{S_{q}(t)\right\}_{t \geq 0}$. Since $S_{q}(t)$ is strongly continuous on $[0, \infty)$, by the uniformly bounded theorem, there exists a constant $M>0$ such that $\left\|S_{q}(t)\right\|_{L(X)} \leq M$ for $t \in[0, b][14]$.

Consider the following fractional partial integrodifferential equation with infinite delay and control in the following form:

$$
\begin{gathered}
{ }^{c} D_{t}^{q}\left[x(t, y)+\int_{-\infty}^{t} a(t, y, s-t) Q_{1}(x(s, y)) d s\right] \\
=\frac{\partial^{2}}{\partial y^{2}}\left[x(t, y)+\int_{-\infty}^{t} a(t, y, s-t) Q_{1}(x(s, y)) d s\right] \\
+\frac{1}{\Gamma(1-q)} \int_{0}^{t}(t-s)^{-q} \\
\times\left[\mu(s, y)+\int_{-\infty}^{s} K(s, x, \xi-s)\right. \\
\times Q_{2}(x(\xi, y)) d \xi \\
\left.+\int_{0}^{s} g(\xi, s) e^{-x(\xi, y)} d \xi\right] d s
\end{gathered}
$$$$
t \in J=[0,1], y \in[0, \pi], t \neq t_{k},
$$

with $x(t, 0)=x(t, \pi)=0, x(t, y)=\phi(t, y), t \in(-\infty, 0], y \in$ $[0, \pi], \Delta x\left(t_{i}\right)(y)=\int_{-\infty}^{t_{i}} q_{i}\left(t_{i}-s\right) x(s, y) d s$, and $y \in[0, \pi]$, 
where $\partial_{t}^{q}$ is the Caputo fractional partial derivative of order $0<q<1 ; \mu:[0,1] \times[0, \pi] \rightarrow[0, \pi]$ is continuous and in $t ; q_{i}: R \rightarrow R$ are continuous; $0<t_{1}<t_{2}<\cdots<t_{n}<b$ are prefixed numbers; and $\phi \in \mathscr{B}_{h}$.

Let $h(s)=e^{2 s}, s<0$, then $l=\int_{\infty}^{0} h(s) d s=1 / 2$. Let $\mathscr{B}_{h}$ be the phase space endowed with the norm $\|\varphi\|_{\mathscr{B}_{h}}=$ $\int_{-\infty}^{0} h(s) \sup _{\theta \in[s, 0]}\|\varphi(\theta)\|_{L^{2}} d s$. Let $x(t)(y)=x(t, y)$, and define the bounded linear operator $B: U \rightarrow X$ by $(B u)(t)(y)=$ $\mu(t, y), 0 \leq y \leq \pi, g(t, \phi)(y)=\int_{-\infty}^{0} a(t, y, \theta) Q_{1}(\phi(\theta)(y)) d \theta$, $I_{k}\left(x\left(t_{i}^{-}\right)\right)(y)=\int_{-\infty}^{t_{i}} q_{i}\left(t_{i}-s\right) x(s, y) d s$, and $f(t, \phi, H x(t))(y)=$ $\int_{-\infty}^{0} K(t, y, \theta) Q_{2}(\phi(\theta)(y)) d \theta+H x(t)(y)$, where $H x(t)(y)=$ $\int_{0}^{t} g(s, t) e^{-x(s, y)} d s$ and $\phi(\theta)(y)=\phi(\theta, y),(\theta, y) \in(-\infty, 0] \times$ $[0, \pi]$. Moreover, the linear fractional control system corresponding to (44) is exactly controllable. Further, if we impose suitable conditions on $a, K, Q_{1}, Q_{2}, g, q_{i}$, and $B$ to verify assumptions on Theorem 8 , then the system (44) can be written in the abstract form of (1). Therefore, all the conditions of Theorem 8 are satisfied, and hence, the nonlinear fractional control system (44) is exactly controllable on $[0, b]$.

\section{Acknowledgment}

The work of Yong Ren is supported by the National Natural Science Foundation of China (no. 11371029).

\section{References}

[1] P. Muthukumar and P. Balasubramaniam, "Approximate controllability of mixed stochastic Volterra-Fredholm type integrodifferential systems in Hilbert space," Journal of the Franklin Institute, vol. 348, no. 10, pp. 2911-2922, 2011.

[2] Y. K. Chang, J. J. Nieto, and W. S. Li, "Controllability of semilinear differential systems with nonlocal initial conditions in Banach spaces," Journal of Optimization Theory and Applications, vol. 142, no. 2, pp. 267-273, 2009.

[3] J. Klamka, "Constrained controllability of semilinear systems with delays," Nonlinear Dynamics of Nonlinear Dynamics and Chaos in Engineering Systems, vol. 56, no. 1-2, pp. 169-177, 2009.

[4] J. Klamka, "Stochastic controllability of systems with multiple delays in control," International Journal of Applied Mathematics and Computer Science, vol. 19, no. 1, pp. 39-47, 2009.

[5] J. Klamka, "Local controllability of fractional discrete-time semilinear systems," Acta Mechanica et Automatica, vol. 15, pp. 55-58, 2011.

[6] J. Klamka, "Constrained exact controllability of semilinear systems," Systems \& Control Letters, vol. 47, no. 2, pp. 139-147, 2002.

[7] J. Dabas, A. Chauhan, and M. Kumar, "Existence of the mild solutions for impulsive fractional equations with infinite delay," International Journal of Differential Equations, vol. 2011, Article ID 793023, 20 pages, 2011.

[8] J. Wang, M. Feckan, and Y. Zhou, "Relaxed controls for nonlinear fractional impulsive evolution equations," Journal of Optimization Theory and Applications, vol. 156, no. 1, pp. 13-32, 2013.

[9] M. Haase, The Functional Calculus for Sectorial Operators, vol. 169 of Operator Theory: Advances and Applications, Birkhäuser, Basel, Switzerland, 2006.
[10] I. Podlubny, Fractional Differential Equations, vol. 198 of Mathematics in Science and Engineering, Academic Press, San Diego, Calif, USA, 1999.

[11] R. P. Agarwal, B. de Andrade, and G. Siracusa, "On fractional integro-differential equations with state-dependent delay," Computers \& Mathematics with Applications, vol. 62, no. 3, pp. 1143-1149, 2011.

[12] G. M. Mophou and G. M. N’Guérékata, "Existence of mild solutions of some semilinear neutral fractional functional evolution equations with infinite delay," Applied Mathematics and Computation, vol. 216, no. 1, pp. 61-69, 2010.

[13] X.-B. Shu, Y. Lai, and Y. Chen, "The existence of mild solutions for impulsive fractional partial differential equations," Nonlinear Analysis: Theory, Methods \& Applications A, vol. 74, no. 5, pp. 2003-2011, 2011.

[14] J. Dabas and A. Chauhan, "Existence and uniqueness of mild solution for an impulsive neutral fractional integro-differential equation with infinite delay," Mathematical and Computer Modelling, vol. 57, no. 3-4, pp. 754-763, 2013.

[15] R. Sakthivel and Y. Ren, "Complete controllability of stochastic evolution equations with jumps," Reports on Mathematical Physics, vol. 68, no. 2, pp. 163-174, 2011.

[16] X.-F. Zhou, J. Wei, and L.-G. Hu, "Controllability of a fractional linear time-invariant neutral dynamical system," Applied Mathematics Letters of Rapid Publication, vol. 26, no. 4, pp. 418-424, 2013.

[17] J. Wang and Y. Zhou, "Complete controllability of fractional evolution systems," Communications in Nonlinear Science and Numerical Simulation, vol. 17, no. 11, pp. 4346-4355, 2012.

[18] S. Kumar and N. Sukavanam, "Approximate controllability of fractional order semilinear systems with bounded delay," Journal of Differential Equations, vol. 251, no. 11, pp. 6163-6174, 2012.

[19] R. Sakthivel, N. I. Mahmudov, and J. J. Nieto, "Controllability for a class of fractional-order neutral evolution control systems," Applied Mathematics and Computation, vol. 218, no. 20, pp. 10334-10340, 2012.

[20] J. Klamka, "Controllability and minimum energy control problem of fractional discrete-time systems," in New Trends in Nanotechnology and Fractional Calculus, D. Baleanu, Z. B. Guvenc, and J. A. T. Machado, Eds., pp. 503-509, Springer, New York, NY, USA, 2010.

[21] A. Debbouche and D. Baleanu, "Controllability of fractional evolution nonlocal impulsive quasilinear delay integrodifferential systems," Computers \& Mathematics with Applications, vol. 62, no. 3, pp. 1442-1450, 2011.

[22] J. K. Hale and S. M. V. Lunel, Introduction to Functional Differential Equations, Springer, Berlin, Germany, 1991.

[23] R. Sakthivel, S. Suganya, and S. M. Anthoni, "Approximate controllability of fractional stochastic evolution equations," Computers \& Mathematics with Applications, vol. 63, no. 3, pp. 660668, 2012.

[24] R. Sakthivel, R. Ganesh, and S. Suganya, "Approximate controllability of fractional neutral stochastic system with infinite delay," Reports on Mathematical Physics, vol. 70, no. 3, pp. 291311, 2012.

[25] Z. Yan, "Approximate controllability of partial neutral functional differential systems of fractional order with statedependent delay," International Journal of Control, vol. 85, no. 8, pp. 1051-1062, 2012.

[26] N. Sukavanam and S. Kumar, "Approximate controllability of fractional order semilinear delay systems," Journal of Optimization Theory and Applications, vol. 151, no. 2, pp. 373-384, 2011. 
[27] N. I. Mahmudov, "Approximate controllability of semilinear deterministic and stochastic evolution equations in abstract spaces," SIAM Journal on Control and Optimization, vol. 42, no. 5, pp. 1604-1622, 2003. 


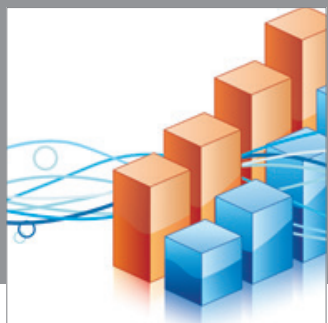

Advances in

Operations Research

mansans

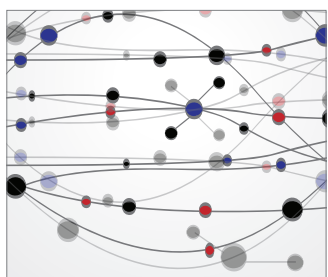

The Scientific World Journal
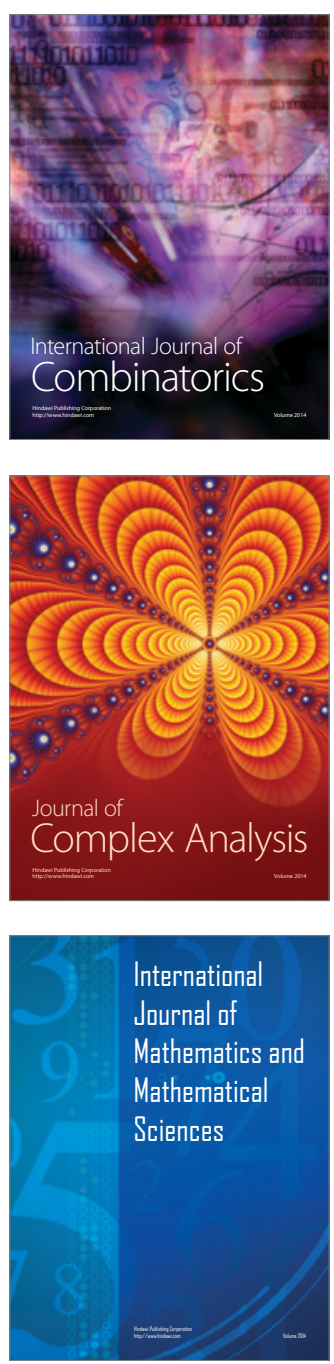
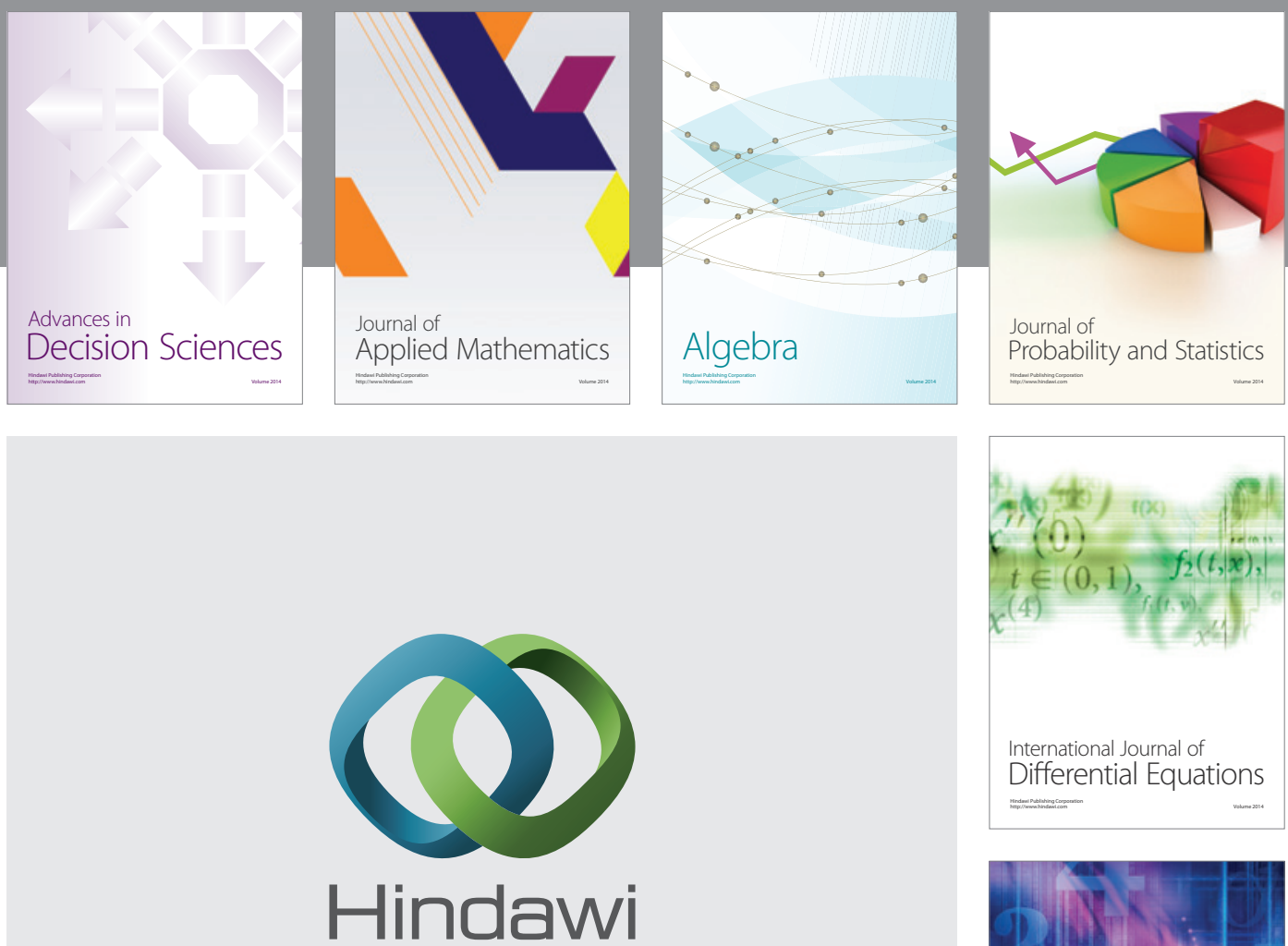

Submit your manuscripts at http://www.hindawi.com
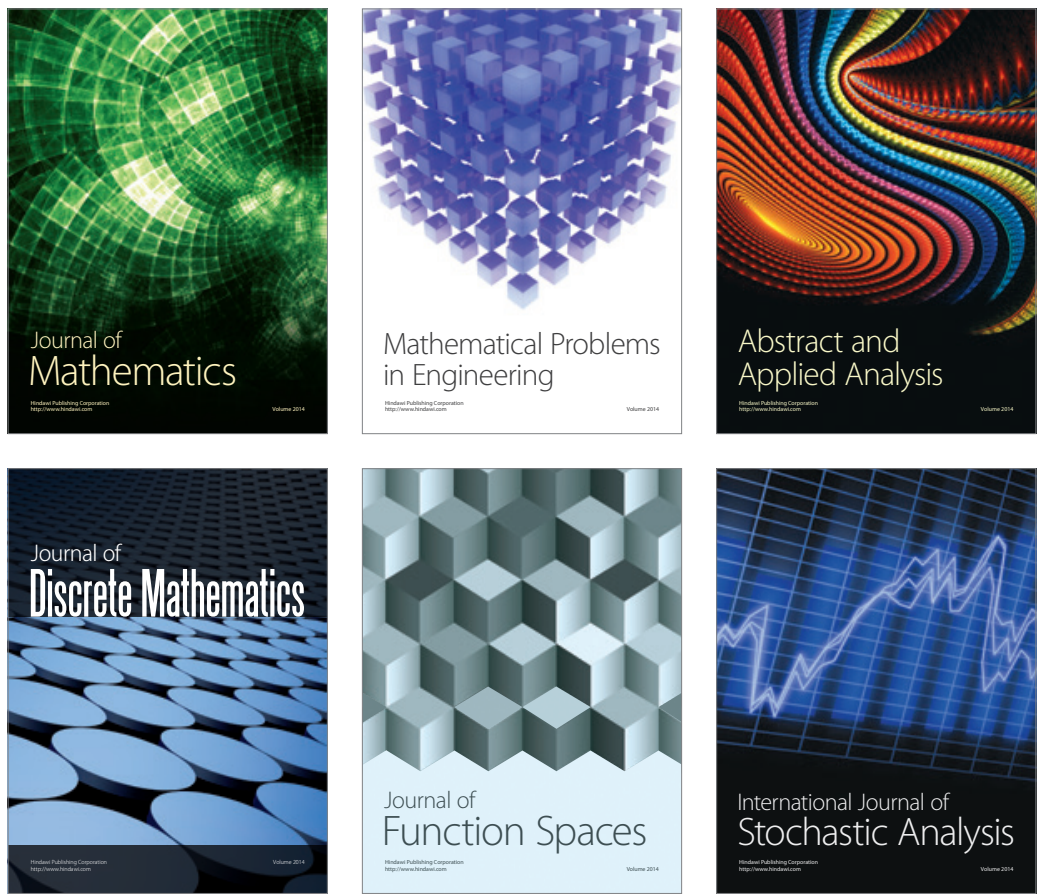

Journal of

Function Spaces

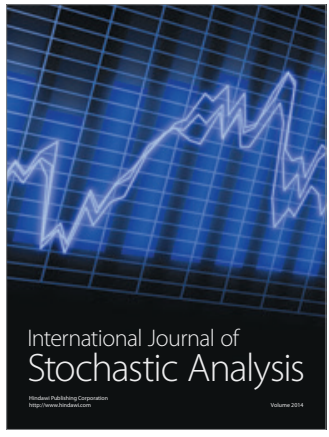

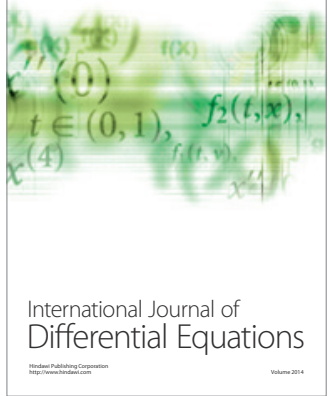
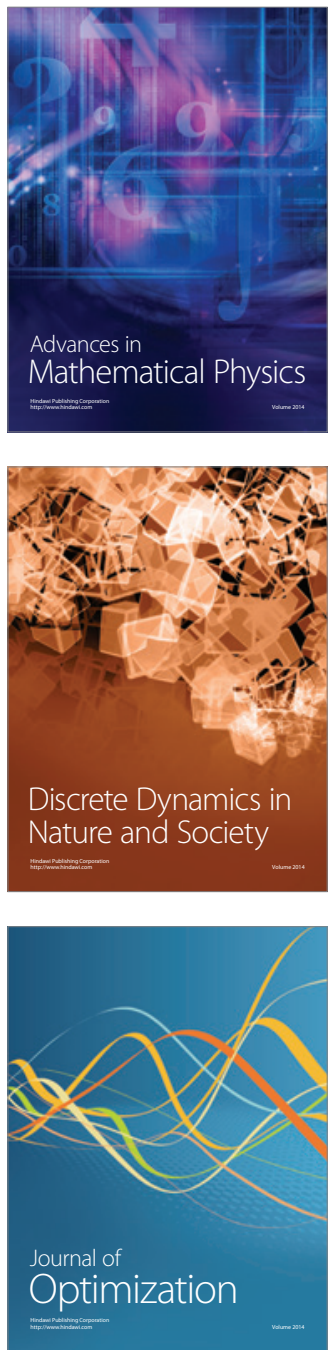